\title{
50 Jahre Randomised Controlled Clinical Trial RCT
}

M. Geiser

1952 erschien im «British Medical Journal» ein Bericht über drei Medical Research Council Trials [1] zur Untersuchung der Chemotherapie bei Lungentuberkulose junger Erwachsener. Dazu wurden fünf Patientengruppen gebildet, um die Wirkung von Bettruhe allein, Streptomycin allein, P.A.S. allein, von Ig Streptomycin plus 5-10 g P.A.S. und von Ig Streptomycin plus $20 \mathrm{~g}$ P.A.S. zu vergleichen. Die zur Prüfung der Wirksamkeit von medizinischen Massnahmen verwendete Methodik ist seither als «Randomised Controlled Clinical Trial» (RCT) in der medizinischen Welt bekannt. 1971 stellte der Epidemiologe A. L. Cochrane eine beunruhigende Inflation im «National Health Service» von Grossbritannien fest, indem laufend kurative und präventive Massnahmen enthusiastisch in die medizinische Praxis eingeführt wurden, bevor Gewissheit darüber bestand, wie wirksam, wirkungslos oder sogar schädlich die Novitäten sind. Cochrane definierte die Effektivität einer medizinischen Massnahme als Fähigkeit, den natürlichen Verlauf einer Krankheit zum Besseren zu wenden. Über die Bedeutung des RCT schrieb Cochrane: «It added the experimental approach to medical research. It opened up a new world of evaluation and control which will, I think, be the key to a rational health service.» [2].

50 Jahre nach der Einführung des RCT und häufigem Einsatz vor allem in der englischsprechenden Welt hat die Inflation im Gesundheitswesen weiter zugenommen, so dass die Notwendigkeit nach objektiver Prüfung der Wirksamkeit von medizinischen Massnahmen noch dringender geworden ist. Es stellt sich heute die Frage, wie es um die Bereitschaft der Akteure im Gesundheitswesen steht, die Effektivität medizinischer Massnahmen möglichst objektiv prüfen zu lassen und die Resultate dieser Prüfungen in die Alltagspraxis umzusetzen, d.h. sie zu respektieren.

1 Diese Tatsache hindert Impfgegner nicht, in der Puls-Sendung vom Fernsehen DRS (am 2. September 2002) aufzutreten und mit irrationalen Argumenten die Laienzuschauer zu verunsichern.

Korrespondenz:

Prof. Dr. med. Max Geiser

Gossetstrasse 49

CH-3084 Wabern

\section{Unterschiedliche Aussagekraft der Effektivitätsprüfungen}

An der Effektivität einer medizinischen Massnahme kann nicht gezweifelt werden, wenn mit einem Medikament eine Krankheit, die unbehandelt eine schlechte Prognose hat (z. B. Tuberkulose, Syphilis, Rachitis usw.), geheilt oder wenn durch einen chirurgischen Eingriff bei einer Verletzung, einem Abszess oder Tumor der ursprüngliche anatomische und funktionelle Zustand wiederhergestellt werden kann. Am wenigsten kann seit mehr als 100 Jahren an der Effektivität von Impfungen gegen Seuchen und Infektionskrankheiten gezweifelt werden ${ }^{1}$. Bei den noch nicht heilbaren Krankheiten gilt es herauszufinden, ob und wie gut eine neue Behandlungsmethode den Verlauf einer Krankheit zum Besseren zu wenden vermag, ob sie gleich viel, mehr oder weniger leistet als bisherige Praktiken.

Bei chirurgischen Eingriffen begnügt man sich meist mit der Feststellung der Resultate in Form einer retrospektiven Kontrolle einer Patientengruppe oder - was schon mehr aussagt mit dem Vergleich einer prospektiv verfolgten mit einer früher kontrollierten Patientengruppe [3]. Der RCT stellt die objektivste Prüfung der Effektivität dar, weil er korrekt durchgeführt die unvoreingenommene Beurteilung der Behandlungsresultate garantiert, Versager und Nebenwirkungen (Risiken) erfasst, die natürliche Regenerations- und Heilungspotenz des menschlichen Organismus und die suggestive Wirkung des Enthusiasmus von Art und Patient (folie à deux!) für die verwendete Methode mit Hilfe des Einsatzes von Placebos zeigt. Korrekt angewendet gilt der RCT als «Goldstandard» der Effektivitätsprüfung von medizinischen Behandlungsmethoden. Es darf jedoch nicht unerwähnt bleiben, dass es wohl nicht schwierig ist, die Randomisierung der Testpersonen $\mathrm{zu}$ realisieren, dass es aber keineswegs leicht ist, die Blindheit der Testperson und des Prüfers sicherzustellen $[4,5]$. Bei gegensätzlichen Resultaten stellt ein einziges negatives Resultat eines methodisch einwandfrei durchgeführten RCT alle RCTs mit positiven Resultaten in Frage.

\section{Wenig Interesse an RTCs im Bereich der Chirurgie, Psychotherapie und Vorsorge}

Angesichts der unvermindert anhaltenden Innovation im Gesundheitswesen ist es heutzutage aus ökonomischen Gründen nicht erwünscht und aus ethischen Gründen nicht zu verantworten, Tausende von Patienten einer Behandlung zu exponieren, die sich nach jahrelangem Ge- 
brauch als effektlos oder sogar schädlich erweist. Während seit Jahrzehnten kein Medikament, abgesehen von sogenannten Naturheilmitteln, auf dem Markt zugelassen wird, das nicht randomisiert gegen ein bereits eingeführtes Medikament oder ein Placebo doppelblind geprüft wurde, werden bei neuen chirurgischen und psychotherapeutischen Behandlungsmethoden und Vorsorgeempfehlungen selten RCTs vor der Einführung durchgeführt $[2,6,7]$. Ein RCT wird oft mit dem Argument unterlassen, dass die neue Methode derart überzeugend sei, dass es aus ethischen und psychologischen Gründen unmöglich sei, einer Gruppe von Patienten die Vorteile der neuen Behandlungsmethode vorzuenthalten. Es gibt aber nicht wenige Beispiele für Behandlungsmethoden, die erst nach längerem Gebrauch mit oder ohne RCT nicht nur als unwirksam, sondern auch als schädlich erkannt wurden [8]. Eines der tragischsten Beispiele ist die Leukotomie, die von 1936 bis 1978 in den USA bei 35000 Patienten gegen Depression, Angst, Phobien und Aggression durchgeführt worden war [9], bis nicht mehr übersehen werden konnte, dass die Schäden weit grösser waren als der Nutzen. Dagegen überlebte die Lugatur der A. mamaria interna zur Behandlung der Angina pectoris nur zwei Jahre, weil sie dank zweier RCTs als Placeboeffekt mit erheblichen Risiken erkannt wurde [5]. Es gibt zahlreiche Beispiele für neue und neueste Technologien, bei denen die Durchführung von RCTs erwünscht wäre, aber bis heute nicht realisiert wurde $[5-8,10,11]$. Dazu gehören endoskopische Eingriffe, künstliche Gelenke, Eingriffe bei degenerativen Veränderungen im Bereiche des Bewegungsapparates. Ferner fehlen RCTs bei vielen Psychotherapien, Kuren und zur Klärung der Indikation von bildgebenden Untersuchungen und von Sinn und Unsinn der Check-ups und vieler Vorsorgeempfehlungen.

Cochrane hat vor 30 Jahren in der Psychiatrie eine wissenschaftliche Renaissance vermisst [2] und beanstandet, dass in der Psychiatrie eine grosse Zahl von Therapien verwendet wird, ohne dass ein Beweis für ihre Wirksamkeit erbracht wurde. Bis in die neueste Zeit liefert die Psychoanalyse ein irritierendes Beispiel für eine Behandlungsmethode, die von Anfang an umstritten war, aber trotz fehlenden Beweises für die klinische Effektivität während eines ganzen Jahrhunderts überzeugte Anhänger nicht nur bei Psychiatern, Psychologen und Sozialarbeitern fand. Sie etablierte sich auch in tonangebenden intellektuellen Kreisen und den Medien als revolutionäre alles erklärende Theorie über die menschliche Natur [13]. Erst am Ende des
20. Jahrhunderts wurden Zweifel an den Behandlungserfolgen im angelsächsischen Sprachraum immer häufiger geäussert [12]. Schliesslich bekam die Psychoanalyse den Ruf einer Glaubenslehre und damit einer Pseudowissenschaft, die ihre breite Akzeptanz als Beweis für ihre Richtigkeit verwendete. Am dritten Weltkongress für Psychotherapie in Wien 2002 wurde Freud von seiner Enkelin Sophie Freud als «falscher Prophet» bezeichnet [12].

\section{Missachtung der Ergebnisse von RTCs in der Komplementär- und Alternativ- medizin (CAM)}

Ähnlich wie bei der Psychoanalyse sind auch bei der Alternativmedizin messianischer Eifer, Personenkult, Dogmatik und Verwendung der Popularität als Beweis für die Richtigkeit zu beobachten. Die Missachtung der negativen Ergebnisse von Effektivitätsprüfungen scheint für die Alternativmedizin charakteristisch zu sein.

Der Widerstand der Alternativ- und sogenannten Naturheiler gegen rigorose Effektivitätsprüfungen wurde kürzlich von E. Ernst in Form von acht Argumenten dargestellt [14], wobei das letzte Argument gegen die negativen Resultate von RCTs «entwaffnend» ist: Die rigorose wissenschaftliche Prüfung mit Hilfe von RCTs sei unsinnig, weil sie zeige, dass die CAM nicht besser ist als Placebos und somit den Patienten vom Gebrauch der CAM-Methoden abhalten werde, obwohl diese dem Patienten helfen, ohne dass die Wissenschaft ihm sage, dass die CAM-Methoden funktionieren.

E. Ernst und Mitarbeiter haben 2001 eine 444 Seiten umfassende Übersicht über diagnostische und therapeutische CAM-Methoden unter Berücksichtigung der in der Literatur fast für jede Methode auffindbaren zahlreichen RCTs und Metaanalysen herausgegeben [15]. Der kritische Leser dieser praktisch die ganze existierende Literatur berücksichtigenden Untersuchung muss nicht unerwartet feststellen, dass den CAM-Methoden ausser der bekannten Placebowirkung entweder keine oder lediglich eine unbedeutenden spezifische Wirkung zugeschrieben werden kann, die konventionellen Methoden unterlegen ist. $\mathrm{Zu}$ diesem Ergebnis passt, dass von den Vertretern der CAM für das gleiche Leiden eine grosse Zahl von verschiedenen alternativen Methoden $^{2}$ empfohlen wird, dass keine Übereinstimmung zwischen den Befürwortern der verschiedenen Systeme besteht und dass sogar eindeutig contraindizierte Methoden empfohlen werden. 
Es besteht kein Zweifel, dass Placebos, vor allem in der ambulanten Heilkunde und in der Selbstbehandlung, eine nicht kleine Rolle spielen. Sie sollten aber wie die bei selbstheilenden alltäglichen Gesundheitsstörungen seit jeher verwendeten «Grossmuttermethoden» in Form von beruhigender Zuwendung, Trost, Tees, Süppchen, Salben und Umschlägen fast nichts kosten. Im Gegensatz zu diesen für jedermann erschwinglichen Hausmittelchen verwenden die Alternativheiler eine fast unbegrenzte Zahl von bizarren Placebos und betreiben damit ein lukratives Geschäft, das eine Käuflichkeit der Gesundheit vortäuscht und bei ernsthaften Leiden falsche Hoffnungen weckt.

Die Tatsache der Unwirksamkeit bei nicht selbstheilenden Krankheiten und die von Ernst [15] ebenfalls erwähnte Tatsache, dass die CAMKonsumenten die alternativen Heilpraktiken zusätzlich und nicht anstelle der konventionellen Medizin verwenden, dass sie vermögend sind, über mehr Bildung verfügen als die Nichtkonsumenten und für die Kosten häufig selbst aufkommen, sprechen dafür, dass die CAM der individuellen Liebhaberei im freien Markt überlassen werden kann. Die CAM braucht keine Unterstützung durch die Standesorganisation und keine Solidarität durch die Nichtkonsumenten. Interessant ist die Angabe, dass die Konsumenten der CAM mehrheitlich weiblich sind. Seit langem bekannt ist die Tatsache, dass Beschwerden im Bereiche des Bewegungsapparates ein Tummelfeld für Alternativheiler darstellen [16].

\section{Popularität und Sicherheitsrisiken der CAM}

$3 \mathrm{Zu}$ den wenig beachteten Risiken gehören das Fehlen einer rigorosen Regulierung pflanzlicher Heilmittel, ihre Verunreinigung mit pathogenen Mikroorganismen, Toxinen, Pestiziden, Herbiziden und Schwermetallen und ihre Vermischung mit pharmazeutischen Wirkstoffen. Bekannter sind die Risiken, die von Heilpraktikern ohne medizinische Qualifikation ausgehen (Impfgegnerschaft, Verhinderung und Verzögerung von lebensrettenden Massnahmen, Missachtung von Kontraindikationen, Veränderung und Abbruch laufender Therapien und mangelnde diagnostische Kompetenz) und durch das Verhalten der CAM-Konsumenten bedingt sind (keine Meldung von ernsthaften Nebenwirkungen, Befolgung von irreführenden Ratschlägen der Gesundheitsliteratur durch ernsthaft kranke Patienten).
Da kein Zweifel darüber besteht, dass die CAM im Gegensatz zur wissenschaftlich überprüften Medizin neben der Placebowirkung keine unverzichtbaren spezifischen Wirkungen auf den Krankheitsverlauf vorweisen kann und mit Sicherheitsrisiken $^{3}$ belastet ist, drängt sich die Suche nach den Ursachen für die trotz bemerkenswerter Effektivität der konventionellen Medizin sogar zunehmende Beliebtheit der alternativen Praktiken auf. Im «Desktop Guide to Complementary and Alternative Medicine» [15] findet sich eine tabellarische Zusammenfassung der vermuteten Ursachen für die Ablehnung der wissenschaftlich fundierten Medizin und für die Beliebtheit der CAM: Abgelehnt wird die gewöhnliche Medizin wegen Enttäuschung des Patienten (Wirkungslosigkeit, Nebenwirkungen, ungenügende Kommunikation mit dem Arzt, Warteliste), bei antiwissenschaftlicher und An-
tiestablishment-Haltung des Patienten, bei Verzweiflung und wegen hoher Kosten. Beliebt sind alternative Heilpraktiken, weil sie mit der Lebensphilosophie des Patienten übereinstimmen (Spiritualismus, Holismus, aktive Rolle des Patienten, intuitive Erklärung der Wirkung, «Natürlichkeit» der Behandlung), weil sie die Kontrolle über die eigene Behandlung gestatten und zu einer guten Beziehung zum Therapeuten führen (gleiche Stufe, Zeit für Diskussion, offen für Emotionen, leichte Zugänglichkeit und Steigerung des Wohlbefindens). Politiker erhoffen von der Integration der CAM in die konventionelle Medizin eine Kosteneinsparung im Gesundheitswesen, obwohl es keine Beweise für eine solche Wirkung gibt [15].

Für die Häufigkeit der Inanspruchnahme der CAM werden für die USA 42\%, Australien 49\%, das Vereinigte Königreich $20 \%$ und für Kontinentaleuropa zwischen 20 und 50\% der Patienten angegeben.

\section{Zusammenfassung}

Der RCT kann nur dann Schlüssel zu einem rationalen Gesundheitswesen werden, wenn diese seit 50 Jahren bekannte Methode der Effektivitätsprüfung medizinischer Massnahmen konsequent und korrekt durchgeführt wird, ihre Resultate respektiert werden und die subjektive Meinung von Patient und Therapeut nicht kritiklos als Beweis für die Wirksamkeit einer Behandlungsmethode gehalten wird im Sinne einer «Opinion-based Medicine» [14].

Für eine undogmatische Medizin, die auf der unvoreingenommenen Erforschung der Natur innerhalb und ausserhalb des Menschen beruht, sind rigorose Effektivitätsprüfungen unverzichtbar. Die Respektierung ihrer Ergebnisse ist bei den Akteuren im Gesundheitswesen ein Zeichen von Seriosität. Der Beitrag des RCT zur Evaluation der Heilkunde und der Heilkundigen und zur Vermeidung von Rückfällen in dogmatische Glaubenslehren ist unverkennbar und $\mathrm{zu}$ begrüssen. Aber die Rückschau auf die 50 Jahre seiner Anwendung und verpassten Anwendung zeigt, dass die Gesundheitswesen auch in den entwickelten Ländern nicht in dem Mass rationalisiert wurden, wie sich dies Cochrane vor 30 Jahren erhofft haben mag. Die Tatsache, dass es keine Grenzen für das Wünschbare gibt, wenn es um Leben und Gesundheit geht, begünstigt das Überleben von dogmatischen Heilslehren. Der Eifer der Politiker, ihren Wählern möglichst viel zur «Gesundheitsförderung» anzubieten und der Drang und Zwang der Medien, Neues und Spek- 
takuläres ungeprüft zu verbreiten und voreilig Erwartungen zu wecken, stellen nur mit Hartnäckigkeit überwindbare Hindernisse für Rationalisierungsbemühungen dar.

Obwohl für die meisten alternativen Behandlungsmethoden RCTs durchgeführt wurden [15] und die allermeisten Resultate negativ, fraglich oder unbedeutend positiv sind, hat dies den Fortbestand und sogar eine Zunahme der Beliebtheit der CAM nicht verhindert. Solange nicht alle Krankheiten eliminiert sind oder mit einfachen Mitteln verhindert oder geheilt werden können, wird es immer enttäuschte Patienten geben, die auf Wunder hoffen und sich durch irrationale Heil- und Verhütungsversprechen irreführen lassen. Deswegen können trotz fehlenden Beweises für Wirksamkeit bizarre dogmatische Heilrituale auch auf dem freien Markt sehr wohl überleben und so gut florieren wie bizarre Produkte im Bereich von Kosmetik, Bekleidung, Schmuck, Gastronomie, Autos, Reisen, Sport und Spiel, die selbstverständlich der individuellen Liebhaberei und Finanzierung überlassen werden. Zum Überleben drängt sich für die CAM keine solidarische Finanzierung auf. Sie speist einen blühenden, aber aus wissenschaftlicher Sicht leerlaufenden Teil des Gesundheits- und Medienmarktes.

Mit weiteren Erfolgen der naturwissenschaftlich fundierten Medizin bei der Verhütung und Heilung von Krankheiten und Unfallfolgen sollte die Reparaturmedizin immer seltener zum Einsatz kommen und dank einer konsequenten Anwendung von Effektivitätsprüfungen weiter rationalisiert werden können. Damit ist denkbar, dass die CAM ihre Beliebtheit im 21. Jahrhundert allmählich verlieren wird und dass zur Befriedigung irrationaler menschlicher Bedürfnisse im Bereich der Gesundheit die bewährten für jedermann erschwinglichen Grossmuttermethoden den Platz der CAM einnehmen werden, wie dies in Arztfamilien seit jeher der Fall war. Auch wenn Ökonomen für die Entdeckung der Rolle der menschlichen Irrationalität im Marktgeschehen (doch wohl eine Binsenwahrheit!) mit dem Nobelpreis geehrt wurden, gibt dies den Akteuren im Gesundheitswesen keinen Freipass, auf Trugschlüsse und Torheiten [5] hereinzufallen. Mitmenschliche, wirksam helfende Zuwendung kann für den ernsthaft kranken Patienten am besten realisiert werden, wenn motivierte, der Vernunft verpflichtete Heilkundige überprüfte Ergebnisse der wissenschaftlichen Erforschung der menschlichen Natur respektieren, sich vom Bluff dogmatischer Heilslehren nicht blenden und von ideologisierten Politikern nicht zu unterwürfigen Leistungserbringern degradieren lassen.

\section{Literatur}

1 Daniels M, Hill AB. Chemotherapy of pulmonary tuberculosis in young adults. An analysis of the combines results of three Medical Research Council trials. Br Med J 1952:1162.

2 Cochrance AL. Effectiveness and Efficiency. Random reflexions on health services. London: The Nuffield Provincial Hospital Trusts; 1972.

3 Rudicel S, Esdaile J. The randomised clinical trial in orthopaedics: obligation or option. J Bone Joint Surg Am 1985;67:1284-97.

4 Leyburn P. A critical look at antidepressant drug trials. Lancet 1967;2(7526):1135-8.

5 Skrabanek P, Mc Cormick J. Follies and Fallacies in Medicine. Glasgow: The Terragon Press; 1989.

6 Stirrat GM, Farrow SC, Fandon J, Dwyer N. The challenge of evaluating surgical procedures. Ann R Coll Surg Engl 1992;74:80-4.

7 Salzmann EW. Is surgery worthwhile? Arch Surg 1985;120:771-6.

8 Geiser M. Wie wissenschaftlich ist die Chirurgie? Schweiz Ärztezeitung 1996;77:318-22.

9 Greenfield S. The Human Brain. London: Weidenfeld und Nicolson; 1997.

10 Goodfellow J. Science and Surgery. J Bone Joint Surg Br 1993;75:345.

11 Gross M. Innovation in surgery. A proposal for phased clinical trials. J Bone Joint Surg Br 1993;75:351-4.

12 Weinberger F. Pseudowissenschaft in der Medizin und der Gesellschaft. Psychoanalyse. Rundbrief 2/02 der Walter-von-Bayer-Gesellschaft für Ethik in der Psychiatrie S. 17-23.

13 Webster R. Why Freud was wrong. Sin, Science and Psychoanalysis. London: Harper Collins Publishers; 1996.

14 Ernst E. What's the point of rigorous research on complementary/alternative medicine? J R Soc Med 2002;95:211-3.

15 Ernst. The Desktop Guide to Complementary and Alternative Medicine. London: Mosby; 2001.

16 Geiser M. Gedanken zur orthopädischen Chirurgie in der zweiten Hälfte des 20. Jahrhunderts. Schweiz Ärztezeitung 2000;81:223-8. 